Article

\title{
Decoding Essential Amino Acid Residues in the Substrate Groove of a Non-Specific Nuclease from Pseudomonas syringae
}

\author{
Lynn Sophie Schwardmann, Sarah Schmitz, Volker Nölle and Skander Elleuche *(D) \\ Miltenyi Biotec B.V. \& Co. KG, Friedrich-Ebert-Straße 68, 51429 Bergisch Gladbach, Germany; \\ 1.schwardmann@web.de (L.S.S.); sarahs@miltenyibiotec.de (S.S.); VolkerN@miltenyibiotec.de (V.N.) \\ * Correspondence: skander.elleuche@miltenyibiotec.de; Tel.: +49-2204-8306-4452
}

Received: 17 October 2019; Accepted: 6 November 2019; Published: 9 November 2019

\begin{abstract}
Non-specific nucleases (NSN) are of interest for biotechnological applications, including industrial downstream processing of crude protein extracts or cell-sorting approaches in microfabricated channels. Bacterial nucleases belonging to the superfamily of phospholipase D (PLD) are featured for their ability to catalyze the hydrolysis of nucleic acids in a metal-ion-independent manner. In order to gain a deeper insight into the composition of the substrate groove of a NSN from Pseudomonas syringae, semi-rational mutagenesis based on a structure homology model was applied to identify amino acid residues on the protein's surface adjacent to the catalytic region. A collection of 12 mutant enzymes each with a substitution to a positively charged amino acid (arginine or lysine) was produced in recombinant form and biochemically characterized. Mutations in close proximity to the catalytic region (inner ring) either dramatically impaired or completely abolished the enzymatic performance, while amino acid residues located at the border of the substrate groove (outer ring) only had limited or no effects. A K119R substitution mutant displayed a relative turnover rate of $112 \%$ compared to the original nuclease. In conclusion, the well-defined outer ring of the substrate groove is a potential target for modulation of the enzymatic performance of NSNs belonging to the PLD superfamily.
\end{abstract}

Keywords: DNase; kinetic profiles; RNase; semi-rational mutagenesis; substrate specificity

\section{Introduction}

Non-specific nucleases (NSN) are a group of enzymes that hydrolyze deoxyribonucleic acid (DNA) and ribonucleic acid (RNA) in all conformations, including single-stranded and double-stranded or linear and circularized substrates, without sequence specificity [1]. NSNs are ubiquitously distributed among all organisms and are of great potential for versatile biotechnological and clinical applications [2-4].

Enzymes that are highly indiscriminate towards different substrates are generally considered as potential evolutionary starting points for developing novel or more specific catalytic activities [5-7]. Members of the phospholipase D (PLD; Enzyme Commission number (EC) 3.1.4.4) superfamily are known to accept a wide range of ester substrates, including nucleic acids [8-10]. PLDs are mainly represented in eukaryotes and predominately catalyze the hydrolysis of phosphatidylcholine to produce choline and phosphatidic acid [11]. Moreover, PLDs act as important key players in various physiological processes, including cell migration and membrane trafficking [10]. This family of enzymes usually encodes two copies of the conserved $\mathrm{HxK}(\mathrm{x})_{4} \mathrm{D}(\mathrm{x})_{6} \mathrm{GSxN}$ motif in one gene.

A structurally related bacterial enzyme (Nuc) has been initially described from the human pathogenic microorganism Salmonella enterica subsp. enterica serovar Typhimurium. Nuc contains a 
single $\mathrm{HxK}(\mathrm{x})_{4} \mathrm{D}(\mathrm{x})_{6} \mathrm{GSxN}$ motif, but forms a homodimer, and is capable of degrading nucleic acids in a non-specific manner [12,13]. This enzyme is among the very few known nucleases that are not dependent on a metal ion in its catalytic region, and is therefore of potential interest for biotechnological applications that take place in buffers supplemented with metal ion chelators, such as ethylenediaminetetraacetic acid (EDTA) or ethylene glycol-bis( $\beta$-aminoethyl ether)- $\mathrm{N}, \mathrm{N}, \mathrm{N}^{\prime}, \mathrm{N}^{\prime}$-tetraacetic acid (EGTA). The group of metal-ion-independent nucleases mainly consists of two PLD-like, site-specific restriction endonucleases from Bacillus firmus and Bacillus megaterium, WSV191 from the white spot syndrome virus and GBSV1-NSN from a thermophilic bacteriophage, as well as the restriction glycosylase R.PabI from the hyperthermophilic archeon Pyrococcus abyssi [14-18].

So far, only three isozymes of bacterial PLD-like NSNs, beside Nuc from S. enterica subsp. enterica serovar Typhimurium, have been investigated and described in detail with regard to their biochemical and biophysical properties: (1) EcNuc from Escherichia coli has been shown to be applicable during cell lysis and protein purification in EDTA-containing buffers; and (2) two isozymes from the plant pathogenic competitor bacterium Pantoea agglomerans were shown to be the result of an ancient gene duplication event followed by diversification $[19,20]$. These enzymes are completely devoid of catalytic performance towards lipids and exclusively degrade nucleic acids in a non-specific manner.

In this study, another metal-ion-independent NSN (DNase/D157G) from Pseudomonas syringae was mutagenized using a semi-rational strategy to gain a deeper insight into the composition of the substrate groove. Homology modeling was applied to identify amino acid residues on the surface of the NSN in the surrounding of the catalytic site, which is buried at the bottom of the putative substrate groove. It has been shown before that positively charged amino acid residues that can interact with the proximal negatively charged phosphate groups in nucleic acids had a stimulating impact on the catalytic activity of human DNase I [21]. Therefore, positively charged amino acids were introduced at positions on the surface of DNase/D157G within the substrate groove. Two regions were defined that were either directly adjacent to the catalytic site (inner ring) or at the border of the substrate groove (outer ring). Substitutions in the inner ring dramatically impaired or completely abolished the catalytic activity, while mutagenesis in the outer ring had no or little effect. DNase variant K119R/D157G displayed increased activity, the temperature optimum of variant S143R/D157G was shifted from $50{ }^{\circ} \mathrm{C}$ to $40{ }^{\circ} \mathrm{C}$, and N95K/D157G and S143K/D157G exhibited an increased tolerance towards $50 \mathrm{mM}$ of EDTA.

\section{Results}

\subsection{Identification of Amino Acid Residues within the Substrate Groove of a NSN from Pseudomonas sp.}

Phylogenetic analyses revealed a highly conserved NSN within the genomes of Pseudomonas species. These enzymes are related to Nuc from S. enterica subsp. enterica serovar Typhimurium ( $\sim 57 \%$ identity in 159 amino acids overlap) and are highly active at neutral $\mathrm{pH}$, in the presence of salt concentrations up to $250 \mathrm{mM}$, and in a temperature range between 4 and $50{ }^{\circ} \mathrm{C}$ (Supplementary Materials Figure S1). It has been shown that amino acid residues D157, E157, and G157 occur naturally in homologues from the genus Pseudomonas. A comparison of the catalytic activities in our laboratory revealed that an enzyme variant containing G157 is superior over a variant with a negatively charged amino acid at position 157 (unpublished results). Therefore, the natural amino acid sequence from a NSN of P. syringae containing a single amino acid substitution at position 157 (DNase/D157G) was used in our study as the starting variant for semi-rational mutagenesis to generate double-mutants.

A homology model of the enzyme DNase/D157G was produced based on the crystal structure from S. enterica subsp. enterica serovar Typhimurium (Figure 1). The enzyme is modelled as a hypothetical homodimer, with the catalytic site buried within a putative substrate groove at the dimeric interface. The catalytic site is composed of amino acids H122, K124, G136, S137, and N139, that are part of the $\mathrm{HxK}(\mathrm{x})_{4} \mathrm{D}(\mathrm{x})_{6} \mathrm{GSxN}$ motif, while D129 has been proposed to be of structural relevance [12]. Fifteen different amino acid residues were identified that are present on the protein surface within the substrate 
groove. These amino acid residues were either assigned to be part of an inner ring that is directly adjacent to the catalytic site or to an outer ring that surrounded the inner ring amino acid residues. The following amino acids were identified as being located on the protein surface close to the catalytic site: Y63, S64, T66, I120, and S141, while P68, H91, G92 D94, N95, A97, A101, K119, A142, and S143 are defined as being part of the outer ring (Figure A1 Appendix A).

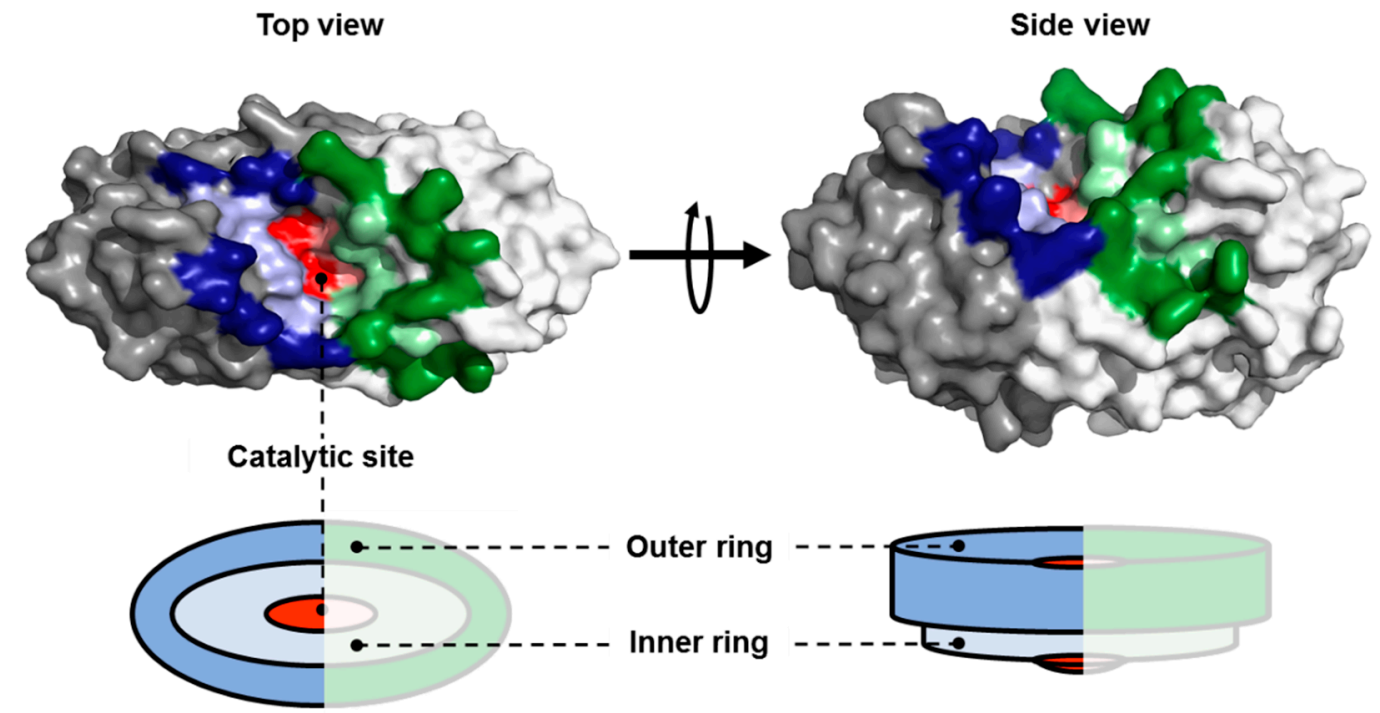

Figure 1. Homology model of non-specific nucleases (NSNs) from Pseudomonas syringae in top and side views. Low resolution model of DNase/D157G using Nuc (PDB: 1BYS_A) as a template. PyMOL was used to highlight a hypothetical dimeric structure with dark and light grey monomers. The conserved residues of the $\mathrm{HxK}(\mathrm{x})_{4} \mathrm{D}(\mathrm{x})_{6} \mathrm{GSxN}$ motif are given in dark and light red. Naturally occurring amino acids of the outer and inner rings are highlighted in dark and light blue, while substituted positively charged amino acid residues are indicated in dark and light green. Cartoon illustrations at the bottom are used to simplify the orientation of amino acid residues of the inner and outer ring within the potential substrate groove surrounding the catalytic site.

Surface-presented amino acid residues were exchanged for lysine or asparagine to improve substrate binding and modulate the enzymatic performance or to identify amino acids essential for catalytic activity. Histidine was not considered due to its bulkiness, aggravating the risk for interference with the structure of the protein. Potential steric hindrance was determined by in silico mutagenesis, and the following substitutions were selected: Y63K, S64K, T66R, P68R, H91R, D94K, N95K, K119R, I120K, S141K, A142R, and S143R. Due to the fact that K119 is the only positively charged amino acid within the substrate groove, this lysine was replaced with asparagine. Amino acid residues G92, A97, and A101 were not mutagenized due to expected clashes with adjacent amino acids.

\subsection{Production and Purification of Recombinant Nuclease Mutants}

Recombinant DNase/D157G double-mutants were produced in Escherichia coli Veggie BL21 (DE3), except for mutant P68R/D157G, because this expression strain could not be transformed with the corresponding expression plasmid. Therefore, Escherichia coli Veggie BL21 (DE3) pLysS was used for the production of this mutant. All recombinant mutant enzymes were produced in soluble form and could be purified using a two-step approach combining affinity and ion exchange chromatography. Purification strategy was optimized using the Äkta purifier (Figure 2). The purification level of all recombinant nucleases was visualized with sodium dodecyl sulfate polyacrylamide gel electrophoresis (SDS-PAGE) and Western blotting analyses using an anti-HIS antibody. 


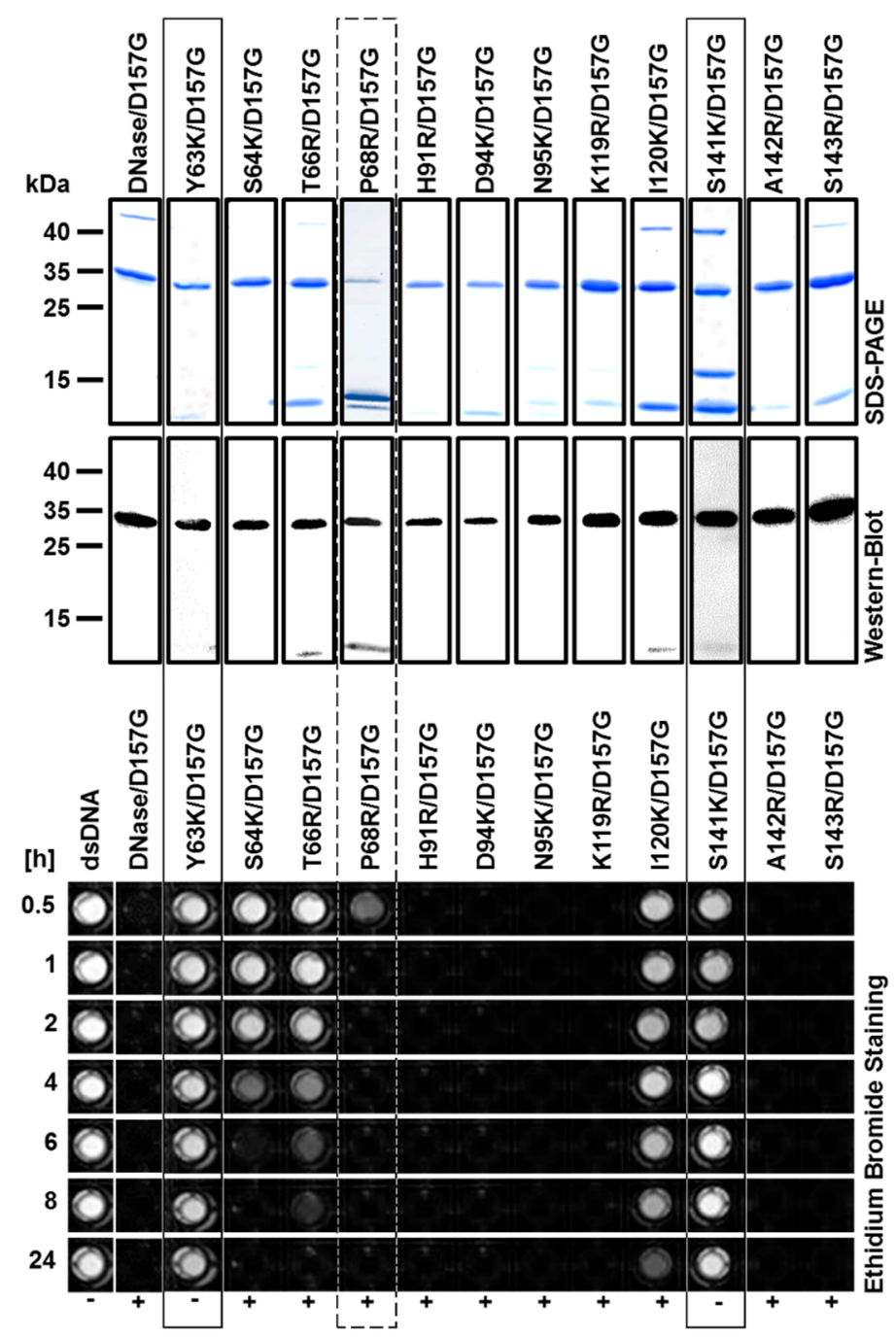

Figure 2. Biochemical and enzymatical analysis of purified nuclease mutants. SDS-PAGE results showed the purity of HIS-tagged nucleases $(29 \mathrm{kDa})$ after a two-step purification approach composed of affinity and ion exchange chromatography (upper row). Western blotting analyses confirmed the presence of HIS-tagged nucleases (middle row). Qualitative activity assays used ethidium bromide staining in 96-well plates (lower row): white colored dots indicate the presence of ethidium bromide intercalating into DNA and grey dots indicate incomplete degradation, while a complete loss of fluorescence is due to complete DNA degradation. Solid line boxes-inactive variants Y63K/D157G and S141K/D157G were purified in non-optimized gravity flow experiments. Dashed line box-variant P68K/D157G was produced in expression strain E. coli Veggie BL21(DE3) pLysS. Note: “+” indicates activity and "-" indicates inactivity. SDS-PAGE results, including all purification steps, are shown in Figure A2 Appendix A.

A qualitative assay using ethidium bromide to visualize non-degraded, double-stranded DNA (dsDNA) was applied to demonstrate that mutants Y63K/D157G and S141K/D157G were inactive, while mutant S64K/D157G completely degraded dsDNA after $6 \mathrm{~h}$, mutant T66R/D157G after $24 \mathrm{~h}$, and mutant I120K/D157G partly hydrolyzes DNA after $24 \mathrm{~h}$. These five mutants contain amino acid substitutions within the inner ring of the substrate groove. The remaining mutants as well as the positive control DNase/D157G completely hydrolyzed dsDNA after 30 min (Figure 2).

To ensure that the correct mutants were purified and characterized, the molecular masses of the purified enzymes were determined by mass spectrometry in addition to sequence verification of generated plasmids. Measured molecular weights are in accordance with predicted masses (Table 1). Variant P68R/D157G could not be properly identified due to impurities. 
Table 1. Molecular masses of nuclease variants.

\begin{tabular}{ccc}
\hline Variant & Theoretical MW (Da) $^{\mathbf{1}}$ & Measured MW (Da) \\
\hline DNase/D157G & $28,900.30$ & $28,900.61$ \\
Y63K/D157G & $28,780.19$ & $28,780.30$ \\
S64K/D157G & $28,856.29$ & $28,856.19$ \\
T66R/D157G & $28,870.28$ & $28,871.01$ \\
P68R/D157G & $28,874.27$ & n.d. \\
H91R/D157G & $28,834.24$ & $28,834.07$ \\
D94K/D157G & $28,828.28$ & $28,828.56$ \\
N95K/D157G & $28,829.26$ & $28,829.40$ \\
K119R/D157G & $28,843.21$ & $28,842.25$ \\
I120K/D157G & $28,830.21$ & $28,829.26$ \\
S141K/D157G & $28,856.29$ & $28,856.10$ \\
A142R/D157G & $28,900.30$ & $28,900.58$ \\
S143R/D157G & $28,884.30$ & $28,884.27$ \\
\hline
\end{tabular}

\subsection{Biochemical Properties of Nucleases}

In vitro activity assays using dsDNA at $25^{\circ} \mathrm{C}$ without the addition of ethidium bromide were conducted to confirm the qualitative plate assays. The reaction was stopped after $1 \mathrm{~h}$ of incubation and samples were loaded onto an agarose gel to investigate the level of hydrolysis of sheared dsDNA (Figure 3). As expected, inactive mutant enzymes Y63K/D157G and S141K/D157G were not capable of degrading dsDNA, while recombinant enzymes S64K/D157G, T66R/D157G, and I120K/D157G, respectively, exhibited reduced activity levels compared to the initial nuclease variant DNase/D157G and the remaining mutants.

\section{dsDNA, sheared}

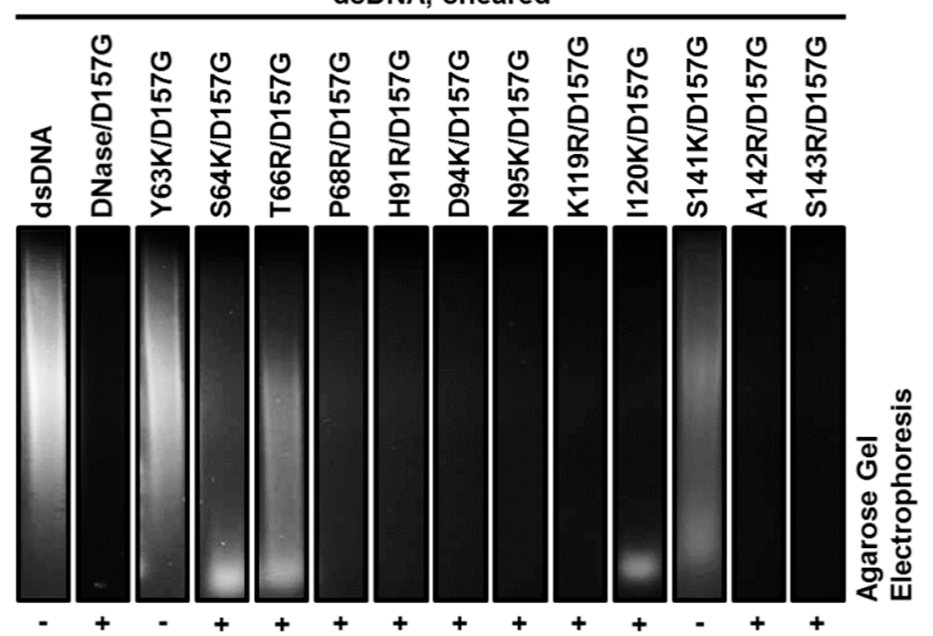

Figure 3. Digestion of sheared dsDNA by nuclease mutants. Sheared dsDNA (UltraPure ${ }^{\mathrm{TM}}$ Salmon Sperm DNA Solution) exhibited an average size of $\leq 2000$ bps. Negative control containing substrate without enzymes is indicated as "dsDNA". The positive control DNase/D157G and mutants S64K/D157G, H91R/D157G, D94K/D157G, N95K/D157G, K119R/D157G, I120K/D157G, A142R/D157G, and S143R/D157G were able to completely hydrolyze sheared dsDNA. S64K/D157G, T66R/D157G, and I120K/D157G partially degraded sheared dsDNA within $1 \mathrm{~h}$ at $25^{\circ} \mathrm{C}$. Y63K/D157G and S141K/D157G did not exhibit activity towards sheared dsDNA. Note: “+" indicates activity and "-" indicates inactivity, without any quantification of the activity level.

After $1 \mathrm{~h}$ of incubation, mutant enzyme T66R/D157G only initiated the hydrolysis of dsDNA with some low molecular weight fragments visible at the bottom of the agarose gel. Therefore, the catalytic activity of this mutant was investigated with regard to its degradation velocity. Identical concentrations 
of mutant enzyme T66R/D157G were incubated with substrate for $30 \mathrm{~min}, 1 \mathrm{~h}, 2, \mathrm{~h}, 4 \mathrm{~h}$, and $24 \mathrm{~h}$, respectively. Reactions were stopped and the samples were loaded onto an agarose gel, revealing that the substrate becomes slowly degraded and is still not completely digested after $4 \mathrm{~h}$ of incubation (Figure 4).

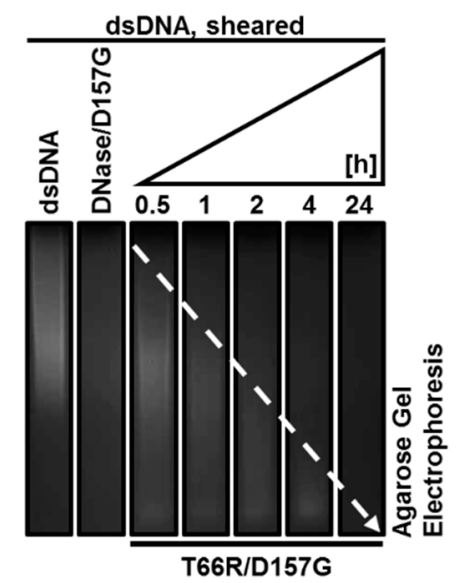

Figure 4. Degradation of sheared dsDNA by mutant T66R/D157G over time. Sheared dsDNA (UltraPureTM Salmon Sperm DNA Solution) exhibited an average size of $\leq 2000$ bps. Reaction was stopped after indicated times (between 0.5 and $24 \mathrm{~h}$ ). White dashed arrow indicates level of nucleic acid molecular weights. The dsDNA is completely hydrolyzed after $24 \mathrm{~h}$ of incubation at $25^{\circ} \mathrm{C}$.

To investigate the substrate promiscuity of active recombinant nuclease mutants, the enzymatic hydrolysis was studied towards the following substrates: unsheared dsDNA, single-stranded genomic DNA (ssDNA), circularized DNA, and RNA from bacteriophage MS2. Mutant enzymes with amino acid substitutions in the outer ring of the substrate groove that were active towards sheared dsDNA (Figure 3) also degraded unsheared dsDNA, ssDNA, circularized DNA, and RNA (Figure 5, Figure A3).

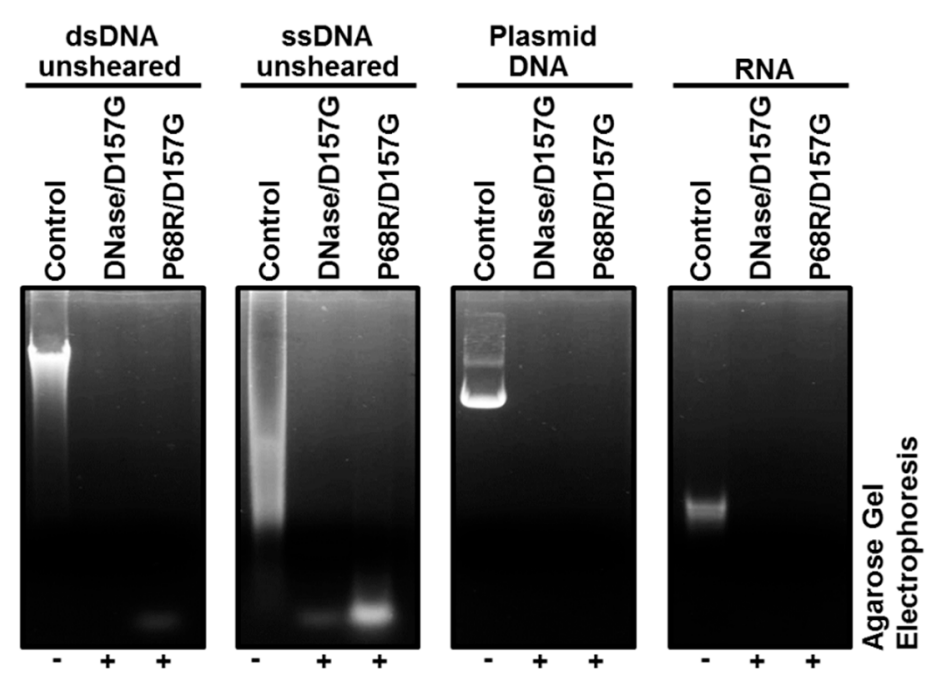

Figure 5. Substrate specificity of mutants with amino acid substitutions in the outer ring. Positive control DNase/D157G and mutant P68R/D157G were incubated for $1 \mathrm{~h}$ at $25^{\circ} \mathrm{C}$ with different types of DNA and RNA. Further outer ring mutants H91R/D157G, D94K/D157G, N95K/D157G, K119R/D157G, A142R/D157G, and S143R/D157G were also able to degrade all types of nucleic acids (Figure A3 Appendix A). "Control" indicates negative controls containing substrate but no enzyme in the reaction mixture.

Nuclease mutant enzyme Y63K/D157G was also inactive towards unsheared dsDNA, ssDNA, and circularized DNA, while S141K/D157G showed some activity on all substrates except sheared 
dsDNA (Figures 3 and 6). However, mutant Y63K/D157G exhibited some activity towards RNA. In good agreement with previous results, mutant enzyme T66R/D157G exhibited reduced activity compared to control DNase/D157G and active mutant enzymes when incubated with both DNA and RNA (Figure 6). In contrast to sheared dsDNA, mutant S64K/D157G completely digested unsheared dsDNA, ssDNA, and circularized DNA, and partially digested RNA, while I120K/D157G was also active on every substrate, but only completely degraded RNA within $1 \mathrm{~h}$ at $25^{\circ} \mathrm{C}$ (Figure 6).
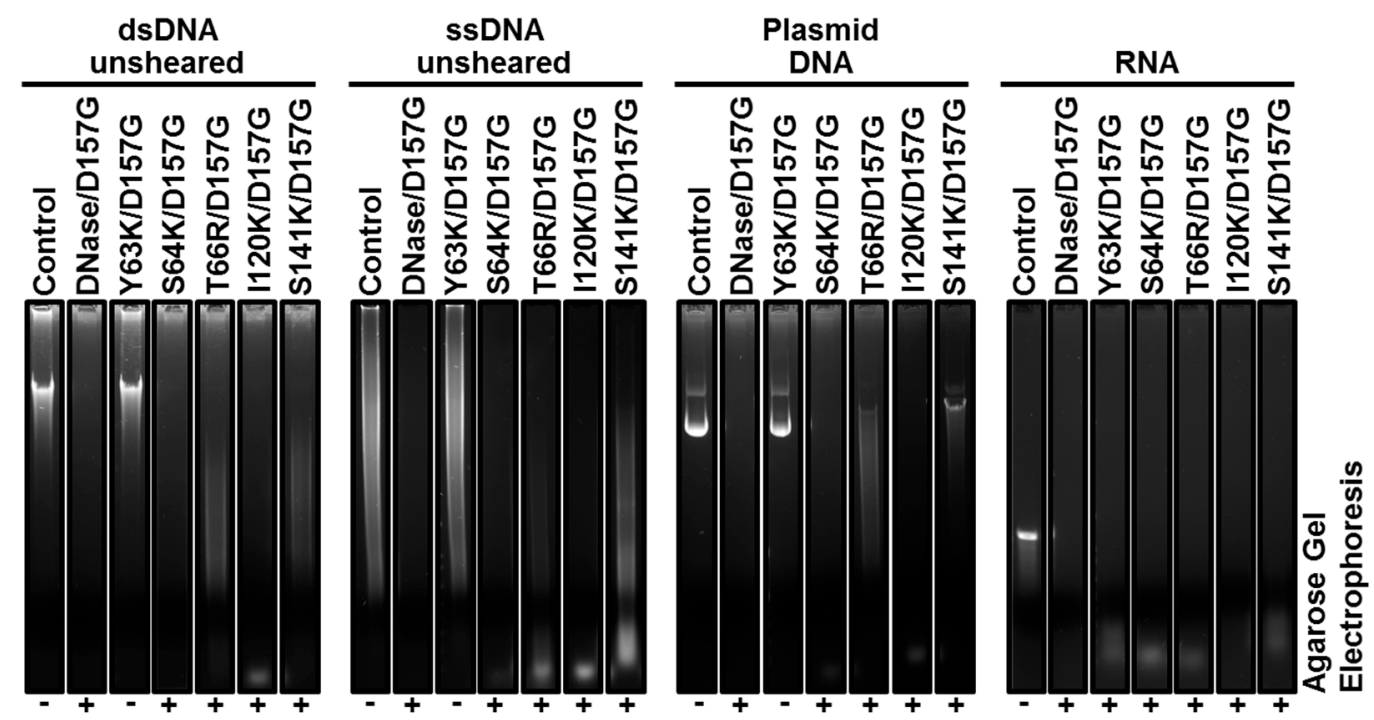

Figure 6. Substrate specificity of mutants with amino acid substitutions in the inner ring. Mutant Y63K/D157G was not able to degrade any type of nucleic acid. Mutants S64K/D157G, T66R/D157G, and I120K/D157G partially degraded all types of nucleic acids within $1 \mathrm{~h}$ at $25^{\circ} \mathrm{C}$, while mutant S141K/D157G exhibited low levels of degradation activity. "Control" indicates negative controls containing substrate but no enzyme in the reaction mixture.

Furthermore, $\mathrm{pH}$ and temperature optima of active mutants were determined. Every mutant as well as the initial variant DNase/D157G displayed optimal activity at $\mathrm{pH}$ 7.0. The temperature optima were around $50^{\circ} \mathrm{C}$ for the initial variant DNase/D157G and all active mutants, except for mutants S64K/D157G and S143R/D157G, which showed optimal activities at $60-70^{\circ} \mathrm{C}$ and $40^{\circ} \mathrm{C}$, respectively.

\subsection{Enzyme Kinetics}

Michaelis-Menten kinetics using sheared dsDNA confirmed the result of the quality activity assays: mutant enzymes Y63K/D157G and S141K/D157G did not exhibited any activity in these assays towards sheared dsDNA and the catalytic performances of mutant enzymes S64K/D157G, T66R/D157G, and I120K/D157G were lower compared to the initial nuclease variant DNase/D157G and remaining active mutant enzymes at $25^{\circ} \mathrm{C}$. Relative turnover rates were below $10 \%$ compared with DNase/D157G and the catalytic efficiency $\left(\mathrm{k}_{\mathrm{cat}} / \mathrm{K}_{\mathrm{M}}\right)$ was below $0.2 \mathrm{~s}^{-1} \mu \mathrm{M}^{-1}$ (Table 2). The turnover number of mutant enzyme K119R/D157G $\left(1034 \mathrm{~s}^{-1}\right)$ exclusively surpassed the catalytic activity of DNase/D157G $\left(924 \mathrm{~s}^{-1}\right)$, but the latter mutant enzyme also exhibited the lowest substrate affinity of all mutants $\left(K_{M}=428 \mu \mathrm{M}\right)$. 
Table 2. Kinetic characteristics of nuclease mutants ${ }^{1}$.

\begin{tabular}{lcccc}
\hline \multicolumn{1}{c}{ Variant } & $\mathbf{K}_{\mathbf{M}}(\boldsymbol{\mu} \mathbf{M})$ & $\mathbf{k}_{\mathbf{c a t}}\left(\mathbf{s}^{-\mathbf{1}}\right)$ & $\mathbf{k}_{\mathbf{c a t}} / \mathbf{K}_{\mathbf{M}}\left(\mathbf{s}^{-\mathbf{1}} \boldsymbol{\mu} \mathbf{M}^{-\mathbf{1}}\right)$ & Relative Turnover Rate $\mathbf{( \% )}$ \\
\hline DNase/D157G & 357 & 924 & 2.58 & 100 \\
Y63K/D157G & n.d. ${ }^{2}$ & n.d. & n.d. & - \\
S64K/D157G & 115 & 8.0 & 0.07 & 0.9 \\
T66R/D157G & 335 & 62 & 0.19 & 6.7 \\
P68R/D157G & 358 & 106 & 0.3 & 11 \\
H91R/D157G & 284 & 290 & 1.02 & 31 \\
D94K/D157G & 224 & 170 & 0.76 & 18 \\
N95K/D157G & 273 & 455 & 1.66 & 49 \\
K119R/D157G & 428 & 1034 & 2.42 & 0.3 \\
I120K/D157G & 115 & 3.0 & 0.03 & - \\
S141K/D157G & n.d. & n.d. & n.d. & 77 \\
A142R/D157G & 352 & 707 & 2.01 & 17 \\
S143R/D157G & 83 & 153 & 1.84 & \\
\hline
\end{tabular}

${ }^{1}$ All experiments were done at $25^{\circ} \mathrm{C} .{ }^{2}$ Not detectable, below the detection limit.

\subsection{EDTA Tolerance of Nuclease Mutants}

As metal-ion-independent proteins, NSNs of the PLD-like family are known to be tolerant in the presence of chelating agents, such as EDTA. DNase/D157G displayed $>80 \%$ relative activity at a concentration of $20 \mathrm{mM}$ EDTA and still preserved $>60 \%$ residual activity in the presence of $50 \mathrm{mM}$ EDTA. Active positively charged mutant enzymes were also tested in the presence of EDTA in concentrations between $1 \mathrm{mM}$ and $50 \mathrm{mM}$, without any abnormalities, except that mutant enzymes P68R/D157G, N95K/D157G, I120K/D157G, and S143K/D157G were almost not affected by any concentration of EDTA (> 80\% residual activity at concentrations up to $50 \mathrm{mM}$ ), while mutant enzymes T66R/D157G and K119R/D157G only showed residual activities of $25 \%$ and $34 \%$ in the presence of $50 \mathrm{~mm}$ EDTA, respectively.

\section{Discussion}

Commercially available NSNs are of high potential for the elimination of nucleic acids during protein downstream processing, to reduce the viscosity, or for prevention of cell clumping in cell sorting approaches $[1,20,22]$. The prototype non-specific nuclease is the metal-ion-dependent NSN from Serratia marcescens that is commercially sold under the trademark "Benzonase ${ }^{\circledR}$ Nuclease" (Merck KGaA, Darmstadt, Germany). This enzyme has been investigated in detail with regard to protein maturation, secretion, catalytic mechanism, and biotechnological applications [2]. Further NSNs, especially metal-ion-independent enzymes, have only been rarely investigated to date $[13,16,18-20,23]$.

In this study, a semi-rational approach was used to select suitable mutation sites within the substrate groove of an NSN from Pseudomonas syringae, which were substituted by site-directed mutagenesis against positively charged amino acid residues to modulate the affinity for negatively charged substrates. Critical amino acid residues for the enzymatic performance of different nucleases were routinely identified by site-directed mutagenesis in previous studies [21,24-26].

Under natural conditions, it has been hypothesized that either positive mutations are first installed in a protein, while negative and neutral mutations are accumulated over time (Neo-Darwinian hypothesis), or neutral mutations pave the way for a flexible evolution and positive or negative mutations are installed as a response to certain conditions (competing hypothesis) [27]. By comparing homology models of proteins with singular amino acid substitutions, it is difficult to determine which of the specific mutations evokes an advantageous, neutral, or even deleterious effect on the protein performance, with certain effects on secondary and tertiary structures often being totally unpredictable [28]. Therefore, in silico mutagenesis was exclusively used to predict steric hindrance between introduced charged amino acid residues and amino acids of the catalytic site, or the predicted substrate groove followed by experimental testing of produced mutants. 
Fifteen amino acid residues on the protein surface near the catalytic site were identified in a NSN that is highly conserved within the genus Pseudomonas. In silico mutagenesis revealed that twelve amino acids could be substituted against either arginine or lysine, without any steric effects in the molecular model. Eleven mutants could be produced in recombinant form in E. coli Veggie BL21 (DE3). However, transformation of E. coli Veggie BL21 (DE3), with an expression plasmid encoding for mutant P68R/D157G, did not result in any clones, but the recombinant enzyme could be produced in expression strain E. coli Veggie BL21 (DE3) pLysS with a very low yield. The additional plasmid pLysS encodes for T7 lysozyme to lower the background expression level of genes under the control of the T7 promoter. Therefore, it can be hypothesized that background expression of the gene-encoding mutant P68R/D157G in pLysS-less expression strains leads to lethality of the host strain. It is worth mentioning that in another experiment, the same results were monitored when proline at position 68 was replaced with negatively charged aspartate in our control. Nevertheless, mutant P68R/D157G only exhibited a turnover rate of $11 \%$ compared with the original variant DNase/D157G. However, the reduced activity may also be dependent on protein impurities that were still present after application of a two-step purification approach.

Artificially increasing the positive charge of the putative substrate groove in the NSN did not accelerate the catalytic performance of the enzyme. In another study, the activity of human DNase I also dropped with the addition of basic amino acids compared with the wild-type enzyme. However, suboptimal conditions for the wild-type enzyme, such as increased salt concentrations, accelerated the performance of the mutated enzyme variants [21]. Eleven out of twelve mutants in our portfolio displayed reduced activity at optimal conditions, while K119R/D157G was the only variant exhibiting an increased turnover number compared with DNase/D157G. However, this enzyme was the sole exception with a basic amino acid replaced by another basic amino acid. Two mutations within the inner ring of the substrate groove, namely Y63K and S141K, completely abolished the ability to hydrolyze sheared dsDNA, while mutant S141K/D157G was still capable of partially hydrolyzing unsheared dsDNA and circular plasmid DNA. Both mutants were also active towards RNA, but it is important to note that although all buffers were prepared under sterile conditions, the possibility that the observed activity is an artefact due to contamination with RNase cannot be excluded. Interestingly, asparagine at position 95 within the outer ring of the substrate groove was slightly impaired with regard to $\mathrm{k}_{\text {cat }}$. A comparable effect has also been observed in another mutant, in which asparagine was replaced by serine. The latter amino acid occurs naturally in the homologous NSN in some members of the genus Pseudomonas.

Mutants were also probed for stability and activity effects in the presence of chelating agents. A slight performance improvement was detected for mutants N95K/D157G and S143K/D157G with regard to the tolerance of high concentrations of EDTA, which were not affected by concentrations up to $50 \mathrm{mM}$. Metal-ion-dependent DNases are usually completely inhibited by low concentrations of EDTA (1-5 mM), and even some metalloproteins that tolerate EDTA are dramatically impaired by concentrations of $50 \mathrm{mM}[29,30]$. However, the related NSNs from Escherichia coli and Pantoea agglomerans were already inhibited at concentrations above $20 \mathrm{mM}$ of EDTA, which is similar to the results obtained with mutant enzymes T66R/D157G and K119R/D157G [19,20]. Nevertheless, these data are in line with the crystal structure of Nuc from S. enterica subsp. enterica serovar Typhimurium, demonstrating that NSNs of the superfamily of PLD proteins are not metal-ion-dependent [12].

The optimal growth temperature of Pseudomonas syringae is at $28^{\circ} \mathrm{C}$, but the highest activity of the nuclease and its mutants was determined to take place between 40 and $70^{\circ} \mathrm{C}$ [31], These results are in line with previous observation of enzymes derived from psychro-, meso-, and thermophiles that displayed a temperature optimum, which is above their preferred growth temperatures $[19,20,29,32,33]$.

Detailed enzyme characterizations are always a prerequisite for understanding the functionality of enzymes and to enable the modulation of their catalytic performances [34]. It has been shown that the enzyme family of NSNs from the genus Pseudomonas is a promising model protein for modifying the catalytic performance with regard to turnover number, temperature optimum, or EDTA tolerance 
by single amino acid substitutions. Due to their enzymatic properties, PLD-like NSNs from bacteria are of great potential for versatile biotechnological applications, and for this reason the discovery of novel enzymes, the optimization of available candidates, and the development of further applications are all highly needed [4,20,35-37]. Finally, the discovery of novel candidates and the extensive characterization in combination with straight-forward protein engineering techniques will lead to the production of more tailor-made enzymes for specific biotechnological applications.

\section{Materials and Methods}

\subsection{Strain and Culture Conditions}

Escherichia coli strains Veggie BL21 (DE3) and Veggie BL21(DE3) pLysS (both from Merck KGaA, Darmstadt, Germany) were used for gene expression and protein production. E. coli strain NEB ${ }^{\circledR} 5$-alpha (New England Biolabs, Frankfurt/Main, Germany) was used for plasmid propagation and maintenance.

\subsection{Computational Sequence Analysis and Structure Modelling}

Protein sequence data of a non-specific nuclease (WP_050543862.1) from the gram-negative, ice-nucleating bacterium Pseudomonas syringae was identified and biochemically characterized in our laboratory (unpublished results). The naturally occurring amino acid substitution D157G in related homologous sequences was shown to be beneficial for catalytic activity of the enzyme compared to the wild-type polypeptide sequence. The three-dimensional model of the bacterial nuclease DNase/D157G was generated by the SWISS-MODEL online server, using the crystal structure of a homologous nuclease (PDB ID:1BYS_A) from Salmonella enterica subsp. enterica serovar Typhimurium as a template. The structure model was analyzed and visualized using the PyMOL software package (PyMOL Molecular Graphics System, Version 2.0 Schrödinger, LLC, New York, NY, USA). Amino acid residues that are located on the surface of the predicted substrate groove were identified and chosen to be substituted against positively charged amino acids (lysine or asparagine). Fifteen amino acid residues were identified to be orientated towards the protein surface close to the catalytic region. These amino acids were assigned to two groups: (1) inner ring (closely located to the catalytic site): Y63, S64, T66, I120, S141, and S143; (2) outer ring (distantly located to the catalytic region): P68, H91, G92, D94, N95, A97, A101, K119, and A142 (Figure A1 Appendix A). In silico mutagenesis was performed to discriminate between lysine and asparagine residue, replacing amino acid residues that are located on the surface of the predicted substrate groove. The substitution with the preferred amino acid residue, either asparagine or lysine, did not result in any steric clashes with adjacent amino acids in the protein for 12 out of 15 amino acids. Therefore, amino acid residues G92, A97, and A101 were excluded from site-directed mutagenesis.

\subsection{Cloning of Nuclease Variants}

Genes-encoding nuclease variants with amino acid substitutions were codon-optimized for expression in E. coli and synthesized by ATUM (Newark, CA, USA). Flanking NcoI and AatII restriction sites were used for unidirectional ligation into linearized vector pET24d(+) (Merck KGaA, Darmstadt, Germany), equipped with a double HIS tag. Sequence verification of inserted genes was done by Eurofins Genomics (Ebersberg, Germany).

\subsection{Gene Expression and Protein Purification}

Expression of genes in E. coli Veggie BL21 (DE3) was performed as described earlier [20]. In brief, nuclease variants were produced with E. coli Veggie BL21(DE3) in $1 \mathrm{~L}$ cultures in $2 \mathrm{~L}$ Erlenmeyer shaking flasks. It was not possible to transform E. coli Veggie BL21 (DE3) with a plasmid coding for the P68K/D157G mutant in our control. Therefore, E. coli BL21 (DE3) pLys was used as an alternative expression host for this NSN variant. Cells were grown under constant shaking $(250 \mathrm{rpm})$ at $37^{\circ} \mathrm{C}$ until an optical density at $600 \mathrm{~nm}\left(\mathrm{OD}_{600}\right)$ of $0.6-0.8$ was reached. Afterwards, gene expression was induced 
by the addition of $0.4 \mathrm{mM}$ isopropyl $\beta$-d-1-thiogalactopyranoside (IPTG). Cells were harvested $4 \mathrm{~h}$ post-induction by centrifugation for $15 \mathrm{~min}$ at $4{ }^{\circ} \mathrm{C}$ and $2880 \times g$.

Cells were disrupted by high-pressure homogenization (constant cell disruption systems, Constant Systems Limited, Northants, UK) at $5{ }^{\circ} \mathrm{C}$ and 1250 bar. Pelleted cells were dissolved in lysis buffer (50 $\mathrm{mM} \mathrm{NaPO}_{4}, \mathrm{pH} 7.3$ ): $1 \mathrm{~g}$ per $5 \mathrm{~mL}$ with a minimal volume of $20 \mathrm{~mL}$. Crude protein extract was incubated for $30 \mathrm{~min}$ at $37^{\circ} \mathrm{C}$ to enable digestion of nucleic acids by the recombinantly expressed nuclease. Afterwards, the sample was centrifuged at $4000 \times g$ for $30 \mathrm{~min}$ at $4{ }^{\circ} \mathrm{C}$. HIS-tagged fusion enzymes were purified in a two-step approach using a combination of affinity (AC) and ion-exchange chromatography (IEX). Initially, gravity flow experiments using Ni sepharose 6 Fast Flow and SP Sepharose Fast Flow cation exchange chromatography resins (both GE Healthcare, Munich, Germany) were done to test activity of partly purified mutant enzymes. Afterwards, the Äkta purifier (GE Healthcare, Munich, Germany) was used to optimize the purification strategy. Supernatant from cell disruption was loaded onto a HisTrap FF Crude histidine-tagged protein purification column (GE Healthcare, Munich, Germany) equilibrated with $50 \mathrm{mM} \mathrm{NaPO}_{4}, 50 \mathrm{mM} \mathrm{NaCl}, 5 \mathrm{mM}$ imidazole, $\mathrm{pH}$ 7.3. The loaded column was connected to an Äkta protein purifier system and washed with 10 column volumes of $50 \mathrm{mM} \mathrm{NaPO}_{4}, 50 \mathrm{mM} \mathrm{NaCl}, 50 \mathrm{mM}$ imidazole, $\mathrm{pH}$ 7.3, prior to the elution with $50 \mathrm{mM} \mathrm{NaPO}_{4}, 50 \mathrm{mM} \mathrm{NaCl}, 500 \mathrm{mM}$ imidazole, $\mathrm{pH}$ 7.3. For the purification by IEX, the eluate from the Ni sepharose affinity purification was diluted 1:4 with IEX running buffer $\left(25 \mathrm{mM} \mathrm{NaPO}_{4}, \mathrm{pH}\right.$ 6.0) to reach a conductivity of 7-8 mS/cm. A HiTrap-SP FF column (GE Healthcare, Munich, Germany) was equilibrated with 10 column volumes of IEX running buffer, before the sample was loaded and washed with 10 column volumes of $50 \mathrm{mM}$ sodium phosphate, $300 \mathrm{mM} \mathrm{NaCl}, \mathrm{pH} 6.0$, prior to the elution with $50 \mathrm{mM}$ sodium phosphate, $700 \mathrm{mM} \mathrm{NaCl}, \mathrm{pH}$ 6.0. All steps were performed at a flow velocity of $1 \mathrm{~mL} / \mathrm{min}$. Finally, a PD-10 desalting column (GE Healthcare, Munich, Germany) was used to replace the IEX elution buffer with storage buffer $\left(50 \mathrm{mM} \mathrm{NaPO}_{4}, 25 \mathrm{mM} \mathrm{NaCl}, \mathrm{pH}\right.$ 7.3). SDS-PAGE (ProGel Tris Glycin 4-20\%, Anamed Elektrophorese GmbH, Groß-Bierberau/Rodau, Germany) in combination with a Western blot using a nitrocellulose blotting membrane (GE Healthcare, Munich, Germany) was used for visualization of recombinant nuclease mutants. An anti-HIS horseradish peroxidase (HRP) antibody (Miltenyi Biotec B.V. \& Co. KG, Bergisch Gladbach, Germany) and the Immobilon ${ }^{\text {TM }}$ Western HRP substrate (Merck, Darmstadt, Germany) were used to detect HIS-tagged proteins.

Enzyme concentrations were measured using a (micro-) Bradford approach in 96-well plate format. A plate reader (EMax, Molecular Devices, San Jose, CA, USA) was used to determine absorbances at $590 \mathrm{~nm}$, which were evaluated with the software SoftmaxPro V5 (Molecular Devices, San Jose, CA, USA). The identities of the nuclease mutants based on their molecular masses were verified with the micrOTOF-Q II Benchtop Mass Spectrometer (Bruker, Billerica, MA, USA).

\subsection{Enzyme Activity Assays}

Enzyme activity was determined both qualitatively and quantitatively based on the depolymerization of nucleic acids. All activity assays were done at $\mathrm{pH} 7$, which has been determined to be the optimal $\mathrm{pH}$. Due to the limited stability of nuclease mutants at elevated temperatures, all assays were done at $25^{\circ} \mathrm{C}$. No thermal effect on the stability was observed for any mutant at this temperature. (1) Qualitative ethidium bromide staining: this assay was adopted from [4]. Recombinant enzyme was incubated with $5 \mu \mathrm{g}$ sheared double-stranded genomic DNA (dsDNA), namely UltraPure ${ }^{\mathrm{TM}}$ Salmon Sperm DNA Solution (Thermo Fisher Scientific, Darmstadt, Germany). This method is based on quenching of fluorescence of ethidium bromide intercalated into DNA. Repeated fluorescence recordings were taken using a VWR ${ }^{\circledR}$ imager (VWR international, Radnor, PA, USA) over a duration of up to $24 \mathrm{~h}$. (2) Qualitative visualization by agarose gel electrophoresis: this assay was adopted from [20]. Substrate specificity was tested with sheared dsDNA, unsheared dsDNA, namely deoxyribonucleic acid from calf thymus (Sigma-Aldrich, St. Louis, MO, USA), single-stranded genomic DNA (ssDNA) from calf thymus (Sigma-Aldrich, St. Louis, USA), RNA from bacteriophage MS2 (Sigma-Aldrich, St. Louis, USA), and circularized plasmid DNA. Qualitative levels of activity are exclusively interpreted 
as "+" (active enzyme) and "-" (inactive enzyme) in Figure 3, Figure 5, and Figure 6, and do not allow any quantification of activities. (3) Quantitative measurements were done in Corning ${ }^{\circledR}$ 96-well UV-transparent plates (Merck KGaA, Darmstadt, Germany) using the Victor ${ }^{\mathrm{TM}}$ X4 Multilabel Plate Reader (PerkinElmer, Rodgau, Germany), as described previously [20]. Standard activity assays were conducted in $50 \mathrm{mM}$ sodium phosphate buffer at $\mathrm{pH}$ 7.3. For the generation of $\mathrm{pH}$ profiles, reactions were performed in $50 \mathrm{mM}$ sodium acetate buffer at $\mathrm{pH} 5$ and 6 , in $50 \mathrm{mM}$ sodium phosphate buffer at $\mathrm{pH} 6,7$, and 8, and in Tris/ $\mathrm{HCl}$ buffer at $\mathrm{pH} \mathrm{7,8,} \mathrm{and} \mathrm{9.} \mathrm{Temperature} \mathrm{profiles} \mathrm{were} \mathrm{conducted}$ in the range of 10 to $90{ }^{\circ} \mathrm{C}$. EDTA tolerance was tested at the following concentrations: $0,1,2$, $5,10,20$, and $50 \mathrm{mM}$. (4) Kinetic parameters were determined with $V_{\max }$ and $K_{M}$ obtained from Michaelis-Menten technique by non-linear regression, as described previously [20,38]. It has been speculated that the initial reaction rate of high molecular weight substrates is reduced at high substrate concentrations due to the extension of the lag phase [39]. Therefore, maximum reaction rates were evaluated for each substrate concentration to determine defined kinetic parameters. All experiments were done in triplicate. The error level was below $10 \%$.

\section{Conclusions}

The composition of the substrate groove was investigated by a combination of structural modelling, multiple sequence alignment, and site-directed mutagenesis. Amino acid residues that are in close proximity to the catalytic site (inner ring of the substrate groove) are of tremendous importance for proper activity of NSN, while amino acids at the border of the substrate groove (outer ring) are promising targets for modulation of the enzymatic properties with regard to turnover number, EDTA tolerance, and temperature preference.

\section{Patents}

A patent application describing the utilization of non-specific nucleases from the genus Pseudomonas and their application potential in cell-sorting approaches has been submitted by Miltenyi Biotec B.V. \& Co. KG.

Supplementary Materials: The following are available online at http://www.mdpi.com/2073-4344/9/11/941/s1. Figure S1: Sequence alignment of DNase/D157G vs. Nuc from S. enterica subsp. enterica serovar Typhimurium.

Author Contributions: Conceptualization, S.E.; methodology, L.S.S. and S.S.; validation, L.S.S., S.S., V.N., and S.E.; resources, V.N.; visualization, L.S.S. and S.E.; writing—original draft preparation, S.E.; writing-review and editing, S.E. and V.N.; All authors approved the final manuscript.

Funding: This research received no external funding.

Acknowledgments: The authors thank Jens Hellmer (Miltenyi Biotec B.V. \& Co. KG) for mass spectrometry analyses and Marek Wieczorek (Miltenyi Biotec B.V. \& Co. KG) for discussion.

Conflicts of Interest: The authors declare no conflict of interest. The funders had no role in the design of the study; in the collection, analyses, or interpretation of data; in the writing of the manuscript, or in the decision to publish the results. 


\section{Appendix A}

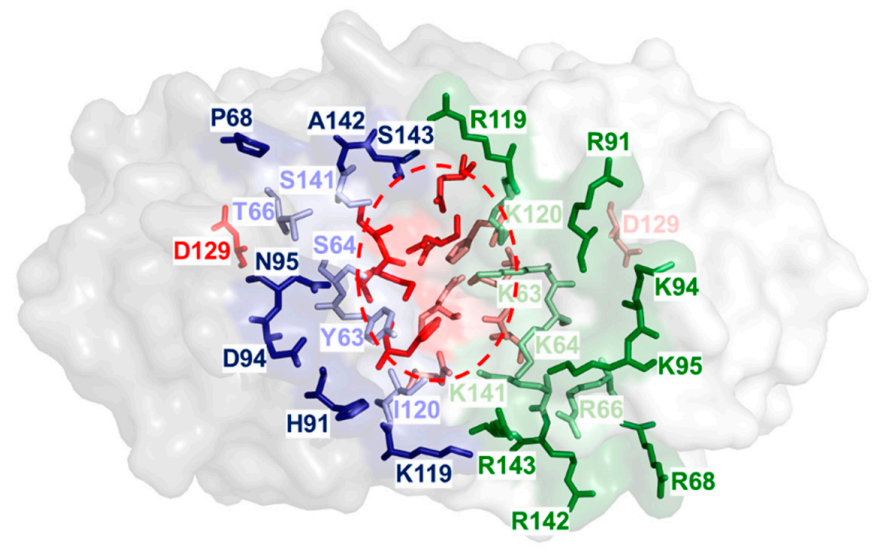

Figure A1. Homology model of NSN from Pseudomonas syringae in top view. Amino acid residues of the conserved $\mathrm{HxK}(\mathrm{x})_{4} \mathrm{D}(\mathrm{x})_{6} \mathrm{GSxN}$ motif are given as sticks in dark and light red in the respective monomer. Numbering of amino acids that are part of the catalytic site was omitted for clarity (encircled in red, dashed line), except for amino acid residue D129, which is part of the $\operatorname{HxK}(x)_{4} \mathrm{D}(\mathrm{x})_{6} \mathrm{GSxN}$ motif, but not part of the catalytic site. Naturally occurring amino acids of the outer and inner rings are also given as sticks and highlighted in dark and light blue, while substituted positively charged amino acid residues are indicated in dark and light green.

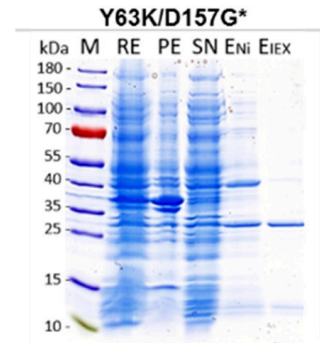

H91R/D157G
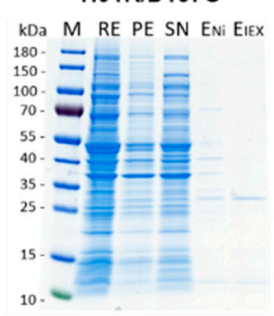$$
\text { 10. }
$$

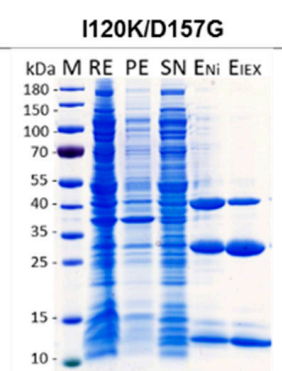

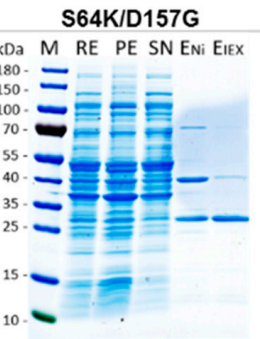

D94K/D157G

kDa M RE PE SN ENi EIEX

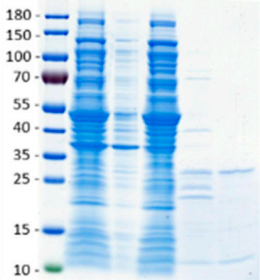

S141K/D157G*
T66R/D157G

KDa M RE PE SN ENi EIEX

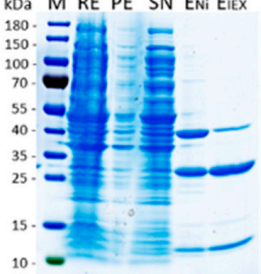

N95K/D157G

kDa M RE PE SN EniEIEX

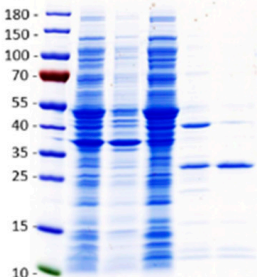

A142R/D157G
P68R/D157G

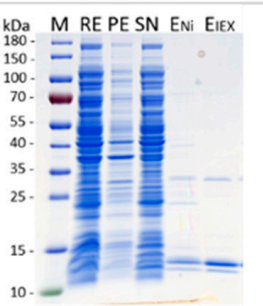

K119R/D157G

kDa M RE PE SN ENi EIEX

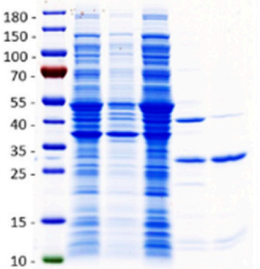

S143R/D157G
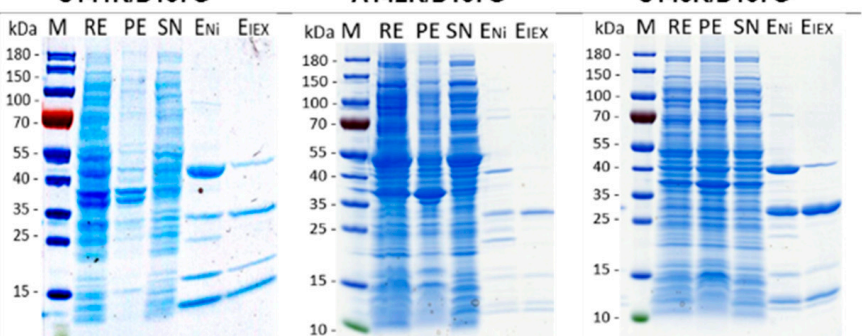

Figure A2. SDS-PAGE results illustrating the purification of all mutant enzymes. M-protein marker, $\mathrm{RE}$ - crude extract, $\mathrm{PE}$ - pellet, $\mathrm{SN}$ - supernatant, $\mathrm{E}_{\mathrm{Ni}}$ - elution fraction Ni-agarose, $\mathrm{E}_{\mathrm{IEX}}$ - elution fraction ion exchange chromatography. Asterisks $\left({ }^{*}\right)$ indicate that inactive mutants Y63K/D157G and S141K/D157G were purified using non-optimized gravity flow purification approaches, resulting in lower purities. 


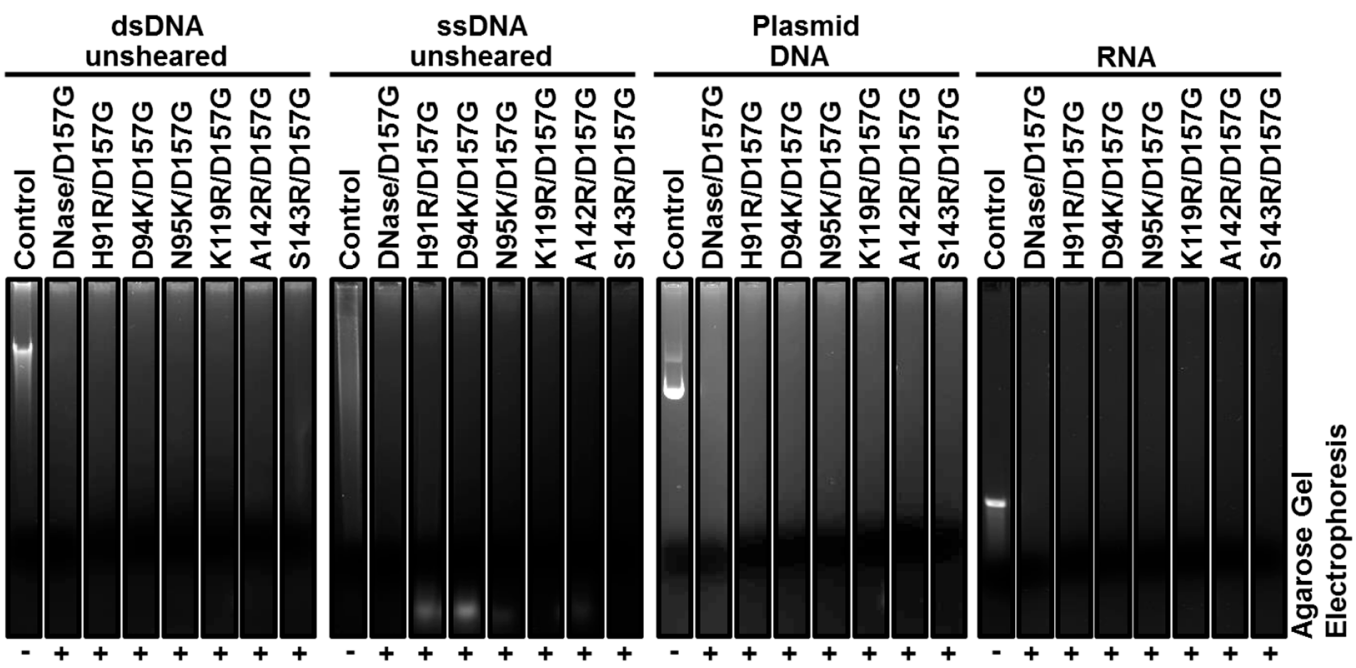

Figure A3. Substrate specificity of mutants with amino acid substitutions in the outer ring. All incubations were done for $1 \mathrm{~h}$ at $25^{\circ} \mathrm{C}$. "Control" indicates negative controls containing substrate but no enzyme in the reaction mixture.

\section{References}

1. Rangarajan, E.S.; Shankar, V. Sugar non-specific endonucleases. FEMS Microbiol. Rev. 2001, 25, 583-613. [CrossRef]

2. Benedik, M.J.; Strych, U. Serratia marcescens and its extracellular nuclease. FEMS Microbiol. Lett. 1998, 165, 1-13. [CrossRef] [PubMed]

3. Dang, G.; Cui, Y.; Wang, L.; Li, T.; Cui, Z.; Song, N.; Chen, L.; Pang, H.; Liu, S. Extracellular sphingomyelinase Rv0888 of Mycobacterium tuberculosis contributes to pathological lung injury of Mycobacterium smegmatis in mice via inducing formation of neutrophil extracellular traps. Front. Immunol. 2018, 9, 677. [CrossRef] [PubMed]

4. Vafina, G.; Zainutdinova, E.; Bulatov, E.; Filimonova, M.N. Endonuclease from gram-negative Bacteria Serratia marcescens is as effective as Pulmozyme in the hydrolysis of DNA in sputum. Front. Pharmacol. 2018, 9, 114. [CrossRef] [PubMed]

5. Khersonsky, O.; Tawfik, D.S. Enzyme promiscuity: A mechanistic and evolutionary perspective. Annu. Rev. Biochem. 2010, 79, 471-505. [CrossRef] [PubMed]

6. Yan, X.; Wang, J.; Sun, Y.; Zhu, J.; Wu, S. Facilitating the evolution of esterase activity from a promiscuous enzyme (Mhg) with catalytic functions of amide hydrolysis and carboxylic acid perhydrolysis by engineering the substrate entrance tunnel. Appl. Environ. Microbiol. 2016, 82, 6748-6756. [CrossRef]

7. Rao, S.J.; Shukla, E.; Bhatia, V.; Lohiya, B.; Gaikwad, S.M.; Kar, A.; Pal, J.K. The Leishmania donovani IMPACT-like protein possesses non-specific nuclease activity. Int. J. Biol. Macromol. 2018, 119, 962-973. [CrossRef] [PubMed]

8. Ponting, C.P.; Kerr, I.D. A novel family of phospholipase D homologues that includes phospholipid synthases and putative endonucleases: Identification of duplicated repeats and potential active site residues. Protein Sci. A Publ. Protein Soc. 1996, 5, 914-922. [CrossRef]

9. Rudolph, A.E.; Stuckey, J.A.; Zhao, Y.; Matthews, H.R.; Patton, W.A.; Moss, J.; Dixon, J.E. Expression, characterization, and mutagenesis of the Yersinia pestis murine toxin, a phospholipase D superfamily member. J. Biol. Chem. 1999, 274, 11824-11831. [CrossRef]

10. Nelson, R.K.; Frohman, M.A. Physiological and pathophysiological roles for phospholipase D. J. Lipid Res. 2015, 56, 2229-2237. [CrossRef]

11. Liscovitch, M.; Czarny, M.; Fiucci, G.; Tang, X. Phospholipase D: Molecular and cell biology of a novel gene family. Biochem. J. 2000, 345, 401-415. [CrossRef] [PubMed]

12. Stuckey, J.A.; Dixon, J.E. Crystal structure of a phospholipase D family member. Nat. Struct. Biol. 1999, 6, 278-284. [CrossRef] [PubMed] 
13. Zhao, Y.; Stuckey, J.A.; Lohse, D.L.; Dixon, J.E. Expression, characterization, and crystallization of a member of the novel phospholipase D family of phosphodiesterases. Protein Sci. 1997, 6, 2655-2658. [CrossRef] [PubMed]

14. Bao, Y.; Higgins, L.; Zhang, P.; Chan, S.H.; Laget, S.; Sweeney, S.; Lunnen, K.; Xu, S.Y. Expression and purification of BmrI restriction endonuclease and its $\mathrm{N}$-terminal cleavage domain variants. Protein Expr. Purif. 2008, 58, 42-52. [CrossRef] [PubMed]

15. Grazulis, S.; Manakova, E.; Roessle, M.; Bochtler, M.; Tamulaitiene, G.; Huber, R.; Siksnys, V. Structure of the metal-independent restriction enzyme BfiI reveals fusion of a specific DNA-binding domain with a nonspecific nuclease. Proc. Natl. Acad. Sci. USA 2005, 102, 15797-15802. [CrossRef] [PubMed]

16. Song, Q.; Zhang, X. Characterization of a novel non-specific nuclease from thermophilic bacteriophage GBSV1. BMC Biotechnol. 2008, 8, 43. [CrossRef] [PubMed]

17. Wang, D.; Miyazono, K.I.; Tanokura, M. Tetrameric structure of the restriction DNA glycosylase R.PabI in complex with nonspecific double-stranded DNA. Sci. Rep. 2016, 6, 35197. [CrossRef]

18. Li, L.; Lin, S.; Yanga, F. Functional identification of the non-specific nuclease from white spot syndrome virus. Virology 2005, 337, 399-406. [CrossRef]

19. Schmitz, S.; Börner, P.; Nölle, V.; Elleuche, S. Comparative analysis of two non-specific nucleases of the phospholipase D family from the plant pathogen competitor bacterium Pantoea Agglomerans. Appl. Microbiol. Biotechnol. 2019, 103, 2635-2648. [CrossRef]

20. Schmitz, S.; Nölle, V.; Elleuche, S. A non-specific nucleolytic enzyme and its application potential in EDTA-containing buffer solutions. Biotechnol. Lett. 2019, 41, 129-136. [CrossRef]

21. Pan, C.Q.; Lazarus, R.A. Hyperactivity of human DNase I variants. Dependence on the number of positively charged residues and concentration, length, and environment of DNA. J. Biol. Chem. 1998, 273, 11701-11708. [CrossRef] [PubMed]

22. Miltenyi, S.; Hübel, T.; Nölle, V. Process for Sorting Cells by Microfabricated Components Using a Nuclease. US Patant 10,018,541 B2, 10 July 2018.

23. Belkebir, A.; Azeddoug, H. Characterization of LlaKI, a new metal ion-independent restriction endonuclease from Lactococcus lactis KLDS4. ISRN Biochem. 2012, 2012, 287230. [CrossRef] [PubMed]

24. Friedhoff, P.; Kolmes, B.; Gimadutdinow, O.; Wende, W.; Krause, K.L.; Pingoud, A. Analysis of the mechanism of the Serratia nuclease using site-directed mutagenesis. Nucleic Acids Res. 1996, 24, 2632-2639. [CrossRef] [PubMed]

25. Zhang, Y.; Li, Z.H.; Zheng, W.; Tang, Z.X.; Shi, L.E. Enzyme activity and thermostability of a non-specific nuclease from Yersinia enterocolitica supsp. palearctica by site-directed mutagenesis. Electron. J. Biotechnol. 2016, 24, 32-37. [CrossRef]

26. Franke, I.; Meiss, G.; Pingoud, A. On the advantage of being a dimer, a case study using the dimeric Serratia nuclease and the monomeric nuclease from Anabaena sp. strain PCC 7120. J. Biol. Chem. 1999, 274, 825-832. [CrossRef] [PubMed]

27. Bommarius, A.S.; Paye, M.F. Stabilizing biocatalysts. Chem. Soc. Rev. 2013, 42, 6534-6565. [CrossRef] [PubMed]

28. Schaefer, C.; Rost, B. Predict impact of single amino acid change upon protein structure. BMC Genom. 2012, 13, S4. [CrossRef]

29. Elleuche, S.; Fodor, K.; Klippel, B.; von der Heyde, A.; Wilmanns, M.; Antranikian, G. Structural and biochemical characterisation of a $\mathrm{NAD}(+)$-dependent alcohol dehydrogenase from Oenococcus oeni as a new model molecule for industrial biotechnology applications. Appl. Microbiol. Biotechnol. 2013, 97, 8963-8975. [CrossRef]

30. Marcal, D.; Rego, A.T.; Fogg, M.J.; Wilson, K.S.; Carrondo, M.A.; Enguita, F.J. Crystallization and preliminary X-ray characterization of 1,3-propanediol dehydrogenase from the human pathogen Klebsiella pneumoniae. Acta Crystallogr. Sect. F Struct. Biol. Cryst. Commun. 2007, 63, 249-251. [CrossRef]

31. Xin, X.F.; Kvitko, B.; He, S.Y. Pseudomonas syringae: What it takes to be a pathogen. Nat. Rev. Microbiol. 2018, 16, 316-328. [CrossRef]

32. Daniel, R.M.; Danson, M.J.; Eisenthal, R.; Lee, C.K.; Peterson, M.E. The effect of temperature on enzyme activity: New insights and their implications. Extremophiles 2008, 12, 51-59. [CrossRef] [PubMed] 
33. Feller, G.; Narinx, E.; Arpigny, J.L.; Zekhnini, Z.; Swings, J.; Gerday, C. Temperature dependence of growth, enzyme secretion and activity of psychrophilic Antarctic bacteria. Appl. Microbiol. Biotechnol. 1994, 41, 477-479. [CrossRef]

34. Erb, T.J. Back to the future: Why we need enzymology to build a synthetic metabolism of the future. Beilstein J. Org. Chem. 2019, 15, 551-557. [CrossRef] [PubMed]

35. Fang, X.J.; Tang, Z.X.; Li, Z.H.; Zhang, Z.L.; Shi, L.E. Production of a new non-specific nuclease from Yersinia enterocolitica subsp. palearctica: Optimization of induction conditions using response surface methodology. Biotechnol. Biotechnol. Equip. 2014, 28, 559-566. [CrossRef] [PubMed]

36. Maciejewska, N.; Walkusz, R.; Olszewski, M.; Szymanska, A. New nuclease from extremely psychrophilic microorganism Psychromonas ingrahamii 37: Identification and characterization. Mol. Biotechnol. 2019, 61, 122-133. [CrossRef] [PubMed]

37. Anisimova, V.E.; Shcheglov, A.S.; Bogdanova, E.A.; Rebrikov, D.V.; Nekrasov, A.N.; Barsova, E.V.; Shagin, D.A.; Lukyanov, S.A. Is crab duplex-specific nuclease a member of the Serratia family of non-specific nucleases? Gene 2008, 418, 41-48. [CrossRef] [PubMed]

38. MacLellan, S.R.; Forsberg, C.W. Properties of the major non-specific endonuclease from the strict anaerobe Fibrobacter succinogenes and evidence for disulfide bond formation in vivo. Microbiology 2001, 147, 315-323. [CrossRef]

39. Friedhoff, P.; Meiss, G.; Kolmes, B.; Pieper, U.; Gimadutdinow, O.; Urbanke, C.; Pingoud, A. Kinetic analysis of the cleavage of natural and synthetic substrates by the Serratia nuclease. Eur. J. Biochem. 1996, 241, 572-580. [CrossRef]

(C) 2019 by the authors. Licensee MDPI, Basel, Switzerland. This article is an open access article distributed under the terms and conditions of the Creative Commons Attribution (CC BY) license (http://creativecommons.org/licenses/by/4.0/). 